\title{
ASCOT simulations of electron energy distribution at the divertor targets in an ASDEX Upgrade H-mode discharge
}

\author{
L K Aho-Mantila ${ }^{1}$, T Kurki-Suonio ${ }^{1}$, A V Chankin ${ }^{2}$, \\ D P Coster $^{2}$ and S K Sipilä ${ }^{1}$ \\ ${ }^{1}$ Helsinki University of Technology, Association Euratom-TEKES, FIN-02015 \\ TKK, Finland \\ 2 Max-Planck-Institut für Plasmaphysik, EURATOM Association, D-85748 \\ Garching, Germany \\ E-mail: leena.aho-mantila@tkk.fi
}

\begin{abstract}
Electron energy distribution at the divertor targets was calculated with the Monte Carlo code ASCOT for an ASDEX Upgrade H-mode discharge. The scrape-off layer plasma background was obtained from the edge fluid code SOLPS. The orbit-following of test particles was performed assuming a fixed Maxwellian plasma background, accounting for the effects of the magnetic geometry, Coulomb interaction with the background, and a prescribed electric potential. The energies recorded from the electrons impinging on the divertor targets indicated that, close to the plates, there should be strong deviations from the thermal Maxwell-Boltzmann distribution. In addition, details of the magnetic geometry and parallel plasma temperature and density profiles were observed to significantly impact the target energy distributions. The obtained discrepancy between the Monte Carlo and fluid results indicates a lack of self-consistency in fluid modelling due to kinetic effects in a medium-collisionality scrape-off layer.
\end{abstract}




\section{Introduction}

The dynamics of a tokamak plasma can be investigated on a large scale by modelling the plasma as a fluid. This approach yields reasonable results provided that the plasma has sufficiently high collisionality for the fluid treatment to be valid. Most of the present-day edge plasma codes such as B2-Eirene [1] and EDGE2D-Nimbus [2, 3] employ the fluid picture. However, due to the low collisionality of the scrape-off layer (SOL), a lot of the crucial edge plasma phenomena may result from kinetic behaviour of the plasma particles that, for one, can not be accounted for by employing fluid dynamics [4]. Complete kinetic modelling presents a tremendous challenge for current edge plasma codes and, for this reason, it is of urgent importance to investigate to which extent the fluid model remains justified in the plasma edge $[5,6]$.

This paper aims at determining whether the assumption of a Maxwell-Boltzmann energy distribution of electrons holds in a medium-collisionality SOL. The orbitfollowing Monte Carlo code ASCOT [7] is employed in simulating the guiding-centre motion of electrons travelling from the outer plasma midplane through an ASDEX Upgrade (AUG) high-confinement mode (H-mode) SOL plasma to the divertor plates. The interaction of the test particles with the fixed plasma background supplied by the SOLPS code [1] is implemented via Monte Carlo operators for small-angle Coulomb collisions, with the underlying assumption of a Maxwell-Boltzmann energy distribution in the plasma background. In addition, the electric potential profile calculated with SOLPS is employed in order to account, to a certain extent, for the SOL parallel electric field. The degree of thermalization of the electron ensemble is estimated by inspecting the energy distributions recorded at the divertor plates, hereafter referred to as target distributions. The results are completed with a detailed investigation of the collisional properties of the electrons along the field lines.

The target distributions obtained with ASCOT are observed to be affected by both the upstream and target background plasma temperatures. In addition, the distribution of energy and particles along the field lines has a significant effect on the thermalization of SOL electrons. Overall, it is observed that the collisionality of the SOL is insufficient for thermalizing all the electrons propagating to the target, and the deviations from the Maxwell-Boltzmann distribution are severe enough to render the fluid model inappropriate for the medium-collisionality edge plasma.

The paper is organized as follows. Section 2 describes the scrape-off layer properties in the H-mode discharge under investigation. In section 3 , the reader is first briefly introduced to the ASCOT simulation environment, and the set-up for the electron simulations is presented. The simulation results are given and discussed here. The conclusions are presented in section 4 .

\section{Hot electrons in H-mode SOL}

H-mode discharges are characterized by the formation of a transport barrier in the plasma edge. Due to the high confinement achieved with the barrier, the core plasma density and pressure are increased from the low-confinement (L-)mode. This produces steep radial density and temperature gradients to the plasma edge, marking out a region known as the plasma pedestal. Outside the pedestal, the SOL has relatively low density. H-mode is typically achieved with divertor tokamaks. In divertor operation, also significant parallel temperature and density gradients are observed in the SOL as a result of ionization of neutrals recycled from the target. With respect to the parallel 


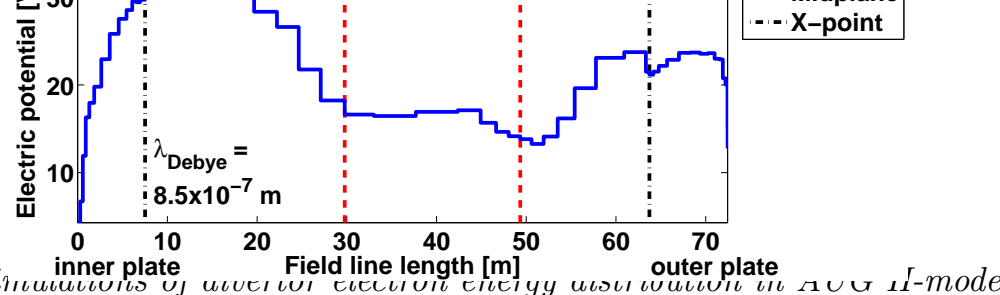

Figure 2. Parallel electric potential profile just outside the separatrix, as calculated by SOLPS. The field line length is measured from the inner divertor plate.

Figure 2 shows the parallel profile of the electric potential just outside the separatrix, as calculated by SOLPS. ASCOT calculates the electric field effects from this prescribed potential, excluding any additional charge imbalance created during the simulations (see [13] and references therein). Calculating the electric field selfconsistently is, unfortunately, beyond the capabilities of ASCOT. The resulting fixed parallel electric field is observed to first accelerate the electrons travelling towards the plates and, only after passing the X-point, become strongly electron-repelling.

In table 1, estimates for the field line -averaged collisionality near the separatrix are given. The numbers describe the collisionality of a suprathermal electron, emerging from the core at the outer midplane (collision frequencies defined in [14]). The SOL collisionality $\nu^{*}$ is defined here as the magnetic connection length, $L_{\mathrm{c}}$, divided by the collisionless mean-free-path, $\lambda_{c}$ (being hence the inverse of the Knudsen number $\left.K=\lambda_{\mathrm{c}} / L_{\mathrm{c}}\right)$. According to the estimates, the electron-ion collisionality is weak especially on the way to the outer divertor plate, and the collisions merely change the pitch of the electron, not the energy. The electron-electron collisionality can, however, be significant with $\nu^{*}$ around 2.9-3.3 on the low-field-side and 5.6-7.7 on the high-field-side.

\section{ASCOT simulations}

\subsection{Description of the ASCOT code}

ASCOT [7] is an orbit-following Monte Carlo code, developed in the 1990s as a collaboration between TKK and VTT (Technical Research Center of Finland). It calculates the guiding-centre orbits of charged particles by integrating the guidingcentre equations of motion over discrete time steps [15]. In between the guiding-centre steps, the velocity components and/or position of the particle are altered by Monte Carlo operators that account for such effects as Coulomb collisions, radio-frequency heating, anomalous transport etc. The Coulomb collision operators, which are the only relevant Monte Carlo operators for the present modelling, are derived from the Fokker-Planck equation assuming non-relativistic field particles with MaxwellBoltzmann energy distribution. Separate collision operators are used for evaluating the change in particle energy and pitch (see detailed description in [15, 16]).

ASCOT employs prescribed magnetic and plasma background data that typically comes from experiments (AUG, JET). The magnetic background data is usually 2dimensional, but even toroidal variation can be taken into account in order to model ripple effects [15]. The $2 \mathrm{D}$ data grid is Cartesian, with $600 \times 600$ grid points. The 
Table 1. Field line -averaged plasma parameters together with various estimates for the collisionality along the flux surfaces of interest. The calculations are performed for an energetic electron with $E=3 k_{\mathrm{B}} T_{\mathrm{omp}}$, where $T_{\mathrm{omp}}$ is the background temperature at the outer midplane, see table 2 .

\begin{tabular}{|c|c|c|c|c|c|c|}
\hline \multirow[b]{2}{*}{ flux surface } & \multicolumn{3}{|c|}{$\begin{array}{l}\text { from omp to } \\
\text { inner target }\end{array}$} & \multicolumn{3}{|c|}{$\begin{array}{l}\text { from omp to } \\
\text { outer target }\end{array}$} \\
\hline & 1.001 & 1.01 & 1.02 & 1.001 & 1.01 & 1.02 \\
\hline \multicolumn{7}{|l|}{ Average parameters: } \\
\hline connection length [m] & 55.2 & 43.9 & 39.9 & 30.8 & 20.1 & 16.7 \\
\hline average electron density $\left[10^{18} \mathrm{~m}^{-3}\right]$ & 34.6 & 11.6 & 6.55 & 37.1 & 10.3 & 6.05 \\
\hline average electron temperature $[\mathrm{eV}]$ & 58.7 & 26.8 & 20.6 & 56.2 & 27.0 & 20.4 \\
\hline average ion density $\left[10^{18} \mathrm{~m}^{-3}\right]$ & 33.8 & 11.3 & 6.16 & 36.4 & 9.88 & 5.61 \\
\hline average ion temperature $[\mathrm{eV}]$ & 96.3 & 66.2 & 61.6 & 90.0 & 71.0 & 63.5 \\
\hline \multicolumn{7}{|l|}{ electron-electron collisions: } \\
\hline mean-free-path, $\lambda_{\mathrm{c}}[\mathrm{m}]$ & 9.9 & 5.7 & 5.4 & 9.3 & 6.4 & 5.8 \\
\hline no. of pitch-changing collisions & 2.7 & 3.7 & 3.5 & 1.6 & 1.5 & 1.4 \\
\hline no. of energy-changing collisions & 2.9 & 4.0 & 3.8 & 1.7 & 1.6 & 1.5 \\
\hline collisionality, $\nu_{\mathrm{ee}}^{*}$ & 5.6 & 7.7 & 7.4 & 3.3 & 3.1 & 2.9 \\
\hline \multicolumn{7}{|l|}{ electron-ion collisions: } \\
\hline mean-free-path, $\lambda_{\mathrm{c}}[\mathrm{m}]$ & 17.6 & 9.7 & 17.5 & 16.6 & 10.9 & 19.1 \\
\hline no. of pitch-changing collisions & 3.1 & 4.6 & 2.3 & 1.9 & 1.8 & 0.9 \\
\hline no. of energy-changing collisions & $<0.01$ & $<0.01$ & $<0.01$ & $<0.01$ & $<0.01$ & $<0.01$ \\
\hline collisionality, $\nu_{\mathrm{ei}}^{*}$ & 3.1 & 4.6 & 2.3 & 1.9 & 1.8 & 0.9 \\
\hline
\end{tabular}

plasma background data is taken either as a flux function $(\rho<1)$ or interpolated into the 2D Cartesian grid $(\rho>1)$. This paper presents the first ASCOT simulations that rely completely on plasma parameters outside the separatrix.

The test particles are simulated with ASCOT until they hit a material structure, such as the vessel wall or divertor target, or when one of the computational end criteria is met. The latter are set by the user as required by the specific problem. In our electron simulations essentially two things can happen: 1) the electron hits the target plate, or 2) the electron is thermalized and becomes part of the SOL bulk plasma. Since there is no reason to follow the latter particles until they diffuse to the wall or divertor, a maximum simulation time of 0.2 seconds is set for each electron. Furthermore, each particle is also assigned a CPU-time maximum of 800 seconds for anomalous situations where a particle might get numerically stuck, for instance, in a grid irregularity. Limiting the CPU-time is of particular importance in these electron simulations, because of the large amount of computation required for calculating the light-particle orbits on open field lines where acceleration of interaction time scales [7] is infeasible.

\subsection{Simulation parameters}

The parallel temperature and density profiles in SOL differ between flux surfaces. For investigating how the various conditions affect the thermalization of a midplane electron ensemble, several initial locations from just outside the separatrix to a centimetre away from it are considered $(\rho=1.001,1.01$ and 1.02). According to table 1 , the electron-electron collisionality should be of the same order on all these flux surfaces. Table 2 shows the relevant background parameters on the midplane and at the target locations. The flux surface just outside the separatrix has, besides the 
Table 2. Background electron parameters on the simulated flux surfaces. 'ot' and 'it' refer to the outer and inner divertor plate, respectively, and 'omp' refers to the initial location at the outer midplane.

\begin{tabular}{lllclll}
\hline$\rho$ & $T_{\mathrm{omp}}[\mathrm{eV}]$ & $T_{\mathrm{it}}[\mathrm{eV}]$ & $T_{\mathrm{ot}}[\mathrm{eV}]$ & $n_{\mathrm{omp}}\left[\mathrm{m}^{-3}\right]$ & $n_{\mathrm{it}}\left[\mathrm{m}^{-3}\right]$ & $n_{\mathrm{ot}}\left[\mathrm{m}^{-3}\right]$ \\
\hline 1.001 & 79.8 & 2.2 & 4.1 & $1.40 \times 10^{19}$ & $2.31 \times 10^{20}$ & $2.34 \times 10^{20}$ \\
1.01 & 34.1 & 6.3 & 12.6 & $8.17 \times 10^{18}$ & $1.94 \times 10^{19}$ & $2.12 \times 10^{19}$ \\
1.02 & 24.7 & 9.9 & 13.8 & $6.09 \times 10^{18}$ & $7.28 \times 10^{18}$ & $1.08 \times 10^{19}$ \\
\hline
\end{tabular}

longest connection length, also the strongest variation of plasma parameters.

The electrons have initially a uniform pitch distribution, corresponding to isotropic velocity distribution. For the initial energy distribution, three cases are considered: $T_{\mathrm{M}-\mathrm{B}}=m T_{\mathrm{omp}}$, where $T_{\mathrm{omp}}$ is the local electron temperature at the point of initialization and $m=1,2,3$. The higher temperatures are used to model situations in which the electron population emerging from inside the separatrix is not thermalized to the local SOL temperature, but carries excess energy from the core. For good statistics of the high-energy tail, the electrons are initialized uniformly in energy up until $10 k_{\mathrm{B}} T_{\mathrm{M}-\mathrm{B}}$, having weight factors given by the Maxwell-Boltzmann distribution.

In order to accurately account for abrupt variations in both SOL background parameters and the motion of the test particles, considerable care is given for adjusting the time step in the electron simulations. The length of the time step $\Delta t$ is restricted by the following criteria: 1) $\Delta t$ should not be longer than $N_{\mathrm{tb}}=1 / 250$ times the bounce time, i.e. the time to complete an orbit (applicable only above X-point). 2) $\Delta t$ must be smaller than $1 / 10$ of the collision time, $t_{\text {coll }}$. 3) During $\Delta t$, the parallel velocity or various drifts do not move the guiding centre poloidally further than 0.05 or $2 \pi N_{\mathrm{tb}}$ times the plasma minor radius, respectively. The latter restriction is rarely needed in the simulations, as the drift velocities for electrons are very low compared to $v_{\text {th }}$.

\subsection{Electron simulations in $S O L$}

3.3.1. Special features in SOL environment The proper working of the ASCOT collision operators was verified by simulating the electrons first on closed field lines. After a sufficiently long simulation time, the electrons propagating in an isothermal plasma with constant density were brought to a Maxwell-Boltzmann energy distribution. Setting then the same conditions to the open field lines and recording the energies at the target plates revealed that the energy of an initially Maxwellian electron ensemble was actually increased during the propagation in SOL. Figure 3 shows the distribution recorded at the target, for an initially thermal ensemble at the outer midplane.

The two observations may seem contradictory at first, but can be explained by considering the simulation set-up. Firstly, the electrons reach the target plates at various time instants. At each poloidal location in SOL, there is a critical parallel velocity above which an electron is likely to be brought directly to the target. The ensemble of electrons launched at a specific instant of time is, hence, constantly depleted by the removal of electrons experiencing a favourable upward shift in energy while propagating through the SOL. Secondly, the depletion of energy is not taken into account in the plasma, where the background temperature remains fixed. Hence, 


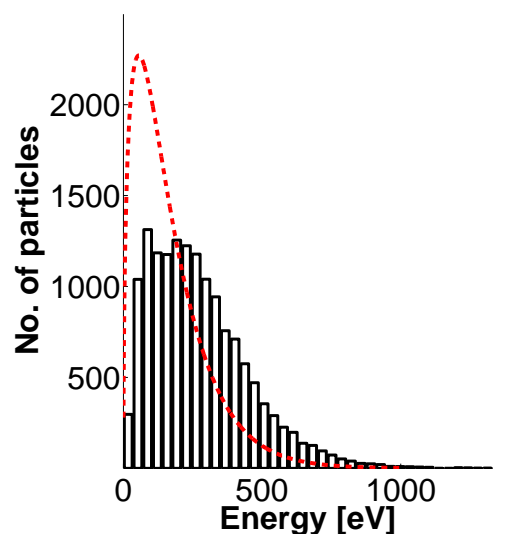

Figure 3. Outer target energy distribution, presented as a histogram (bin width $35 \mathrm{eV}$ ), for thermal electrons initialized at the outer midplane with $\rho=1.02$ and followed through a homogeneous background plasma $\left(n_{\mathrm{e}}=n_{\mathrm{i}}=1.5 \times 10^{19} \mathrm{~m}^{-3}\right.$, $T_{\mathrm{e}}=T_{\mathrm{i}}=111 \mathrm{eV}$ and $E_{\|}=0$.). The background plasma energy distribution is represented by the dashed line. The average energy of the target distribution is $270 \mathrm{eV}$, and the target distribution has a peak around $3 k_{\mathrm{B}} T / 2$.

Table 3. Statistics from the simulation results at $\rho=1.01$ and $\rho=1.02$, when total number of electrons initialized is $N_{\text {omp }}=20000$. All energy values are in electron-volts, and the statistical error in the target energies is $\sim 5 \%$.

\begin{tabular}{llcllll}
\hline$\rho$ & $T_{\mathrm{M}-\mathrm{B}}$ & $E_{\text {omp, ave }}$ & $N_{\text {it }}$ & $N_{\text {ot }}$ & $E_{\text {it,ave }}$ & $E_{\text {ot,ave }}$ \\
\hline \multirow{3}{*}{1.01} & $T_{\text {omp }}$ & 51 & 5589 & 14412 & 25.2 & 44.9 \\
& $2 T_{\text {omp }}$ & 102 & 5652 & 14349 & 26.2 & 46.2 \\
& $3 T_{\text {omp }}$ & 154 & 5618 & 14383 & 30.4 & 52.2 \\
\hline \multirow{3}{*}{1.02} & $T_{\text {omp }}$ & 37 & 4823 & 15178 & 19.6 & 49.3 \\
& $2 T_{\text {omp }}$ & 74 & 4885 & 15116 & 20.0 & 50.4 \\
& $3 T_{\text {omp }}$ & 111 & 4948 & 15053 & 24.0 & 55.5 \\
\hline
\end{tabular}

the cold electrons remaining in the SOL soon replenish the vacant higher energies in the Maxwell-Boltzmann distribution via collisions, and the distribution observed at the target becomes shifted from that of the background. This evident lack of self-consistency in ASCOT results restricts the quantitative analysis, but should not prevent benchmarking the fluid model in the edge.

The effect of several physical mechanisms on the thermalization of SOL electrons was investigated, and the following results were obtained: 1) Excluding test electron collisions with the background ions has practically no effect on the target energy distributions and, hence, the ion temperature and density profiles can be concluded to have a negligible role in the energy distribution of SOL electrons. This is an expected result, as the electron-ion energy exchange has a very low time scale, recall table 1. 2) Including anomalous radial transport with transport coefficient of maximum $1 \mathrm{~m}^{2} / \mathrm{s}$ does not affect the target distributions. 3) Including a fixed parallel electric field in the model increases the simulation times but has a negligible effect on the target distributions. 

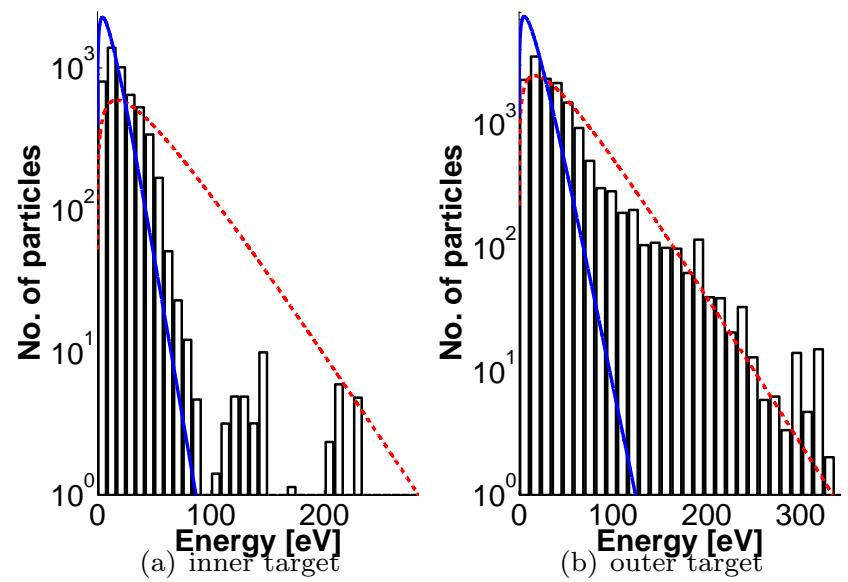

Figure 4. Electron target energy distributions, presented as histograms (bin widths $8.4 \mathrm{eV}$ and $11.6 \mathrm{eV}$ at the inner and outer plate, respectively), on the flux surface $0.5 \mathrm{~cm}$ apart from the outer midplane separatrix, $\rho=1.01$. The solid line represents the background Maxwell-Boltzmann energy distribution at the plate, as predicted by the SOLPS code. The dashed line gives the initial energy distribution of those electrons recorded at the target, corresponding to $T_{\text {omp. }}$.

3.3.2. Target energy distributions at $\rho=1.01-1.02$ The suite of simulations with realistic background parameters shows that only one quarter to one third of the electrons end up on the inner divertor plate, while the rest are brought to the outer plate. This can be concluded to be a direct consequence of the connection lengths to the targets differing roughly by a factor of 2 , recall table 1 . Table 3 shows the statistics of the simulation results for the two outermost flux surfaces, $\rho=1.01-1.02$. The fraction of electrons impinging on the outer target $\left(N_{\mathrm{ot}} / N_{\mathrm{omp}}\right)$ varies somewhat with the flux surface, but does not depend on the initial energy of the distribution. The average target energies are much higher than the divertor temperatures predicted by SOLPS fluid calculations, and they are found to weakly increase with the initial energy of the ensemble. Inner target energies lower than the outer target energies are in accordance with the estimated target electron temperatures, recall table 2.

The target energy distributions at $\rho=1.01$, for the case of thermal initial ensemble, are shown in figure 4. Target distributions at $\rho=1.02$ are similar in form. At the outer target, the distribution is close to Maxwellian with temperature around $T_{\mathrm{omp}}$. A fraction of the electrons seems to have increased their energies on the way to the target, similarly to the case shown in figure 3 . At the inner target, the distribution has a large, almost thermalized component. In addition, there is an excessive number of electrons in the high-energy tail of the distribution. The effect of the hot electron component on the target heat load is, however, small even with initially suprathermal ensembles.

3.3.3. Target energy distributions at $\rho=1.001$ Distinctly different results are obtained for the innermost flux surface with $\rho=1.001$ than for those at the outermost locations. Table 4 shows the statistics of these simulations. The distribution of electrons between the two target plates is again found to be independent of the initial 
Table 4. Statistics from the simulation results at $\rho=1.001$ when total number of electrons initialized is 20000 . A fraction of the electrons $(\approx 10 \%)$ was lost due to the simulation time limitation, but this could be shown not to affect the principal results. All energy values are in electron-volts, and the statistical error in the target energies is $\sim 5 \%$.

\begin{tabular}{lllllrl}
\hline$\rho$ & $T_{\mathrm{M}-\mathrm{B}}$ & $E_{\text {omp, ave }}$ & $N_{\text {it }}$ & $N_{\text {ot }}$ & $E_{\text {it,ave }}$ & $E_{\text {ot,ave }}$ \\
\hline \multirow{3}{*}{1.001} & $T_{\text {omp }}$ & 120 & 5010 & 13136 & 8.6 & 17.3 \\
& $2 T_{\text {omp }}$ & 240 & 5128 & 13069 & 11.3 & 20.9 \\
& $3 T_{\text {omp }}$ & 359 & 4884 & 13333 & 26.2 & 40.1 \\
\hline
\end{tabular}
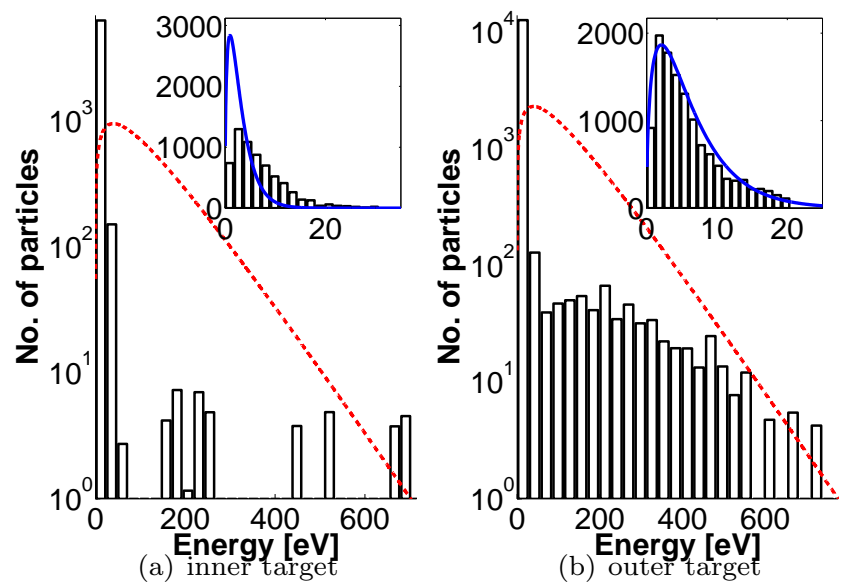

Figure 5. Electron target energy distributions, presented as histograms (bin widths $24.2 \mathrm{eV}$ and $28.4 \mathrm{eV}$ ), on the flux surface closest to the separatrix, $\rho=1.001$. The inset shows a zoom-in to the lowest energies (bin widths $1.2 \mathrm{eV}$ and $1.8 \mathrm{eV}$ ). Representation otherwise as in figure 4 .

Table 5. The total energy of the thermal peak $(E<30 \mathrm{eV}), E_{\mathrm{th}}$, compared to the total energy of the suprathermal electrons $(E>30 \mathrm{eV}), E_{\text {sth }}$, for $\rho=1.001$. 'it' and 'ot' refer to the inner and outer target, respectively. The suprathermal energy portion is significant, increasing with more energetic initial distributions. The thermal energy portion remains constant.

\begin{tabular}{llrllll}
\hline$T_{\mathrm{M}-\mathrm{B}}$ & $E_{\mathrm{it}, \mathrm{th}}$ & $E_{\text {it,sth }}$ & $E_{\text {it,sth }} / E_{\text {it,tot }}$ & $E_{\text {ot,th }}$ & $E_{\text {ot,sth }}$ & $E_{\text {ot,sth }} / E_{\text {ot,tot }}$ \\
\hline$T_{\text {omp }}$ & $35 \mathrm{keV}$ & $8 \mathrm{keV}$ & 0.19 & $66 \mathrm{keV}$ & $161 \mathrm{keV}$ & 0.71 \\
$2 T_{\text {omp }}$ & $35 \mathrm{keV}$ & $23 \mathrm{keV}$ & 0.40 & $66 \mathrm{keV}$ & $207 \mathrm{keV}$ & 0.76 \\
$3 T_{\text {omp }}$ & $32 \mathrm{keV}$ & $96 \mathrm{keV}$ & 0.75 & $64 \mathrm{keV}$ & $470 \mathrm{keV}$ & 0.88 \\
\hline
\end{tabular}

energy of the ensemble. The average target energies, however, increase significantly as more energetic initial ensembles are investigated. At the inner target, for example, the average energy in the $m=3$ case retains the three times higher value than that in the $m=1$ (thermal) case.

Figure 5 shows the target energy distributions at $\rho=1.001$, for the initially thermal case. At both plates, a high thermal peak at low energies corresponding to the divertor temperature is observed. At the outer plate, the thermal peak is 

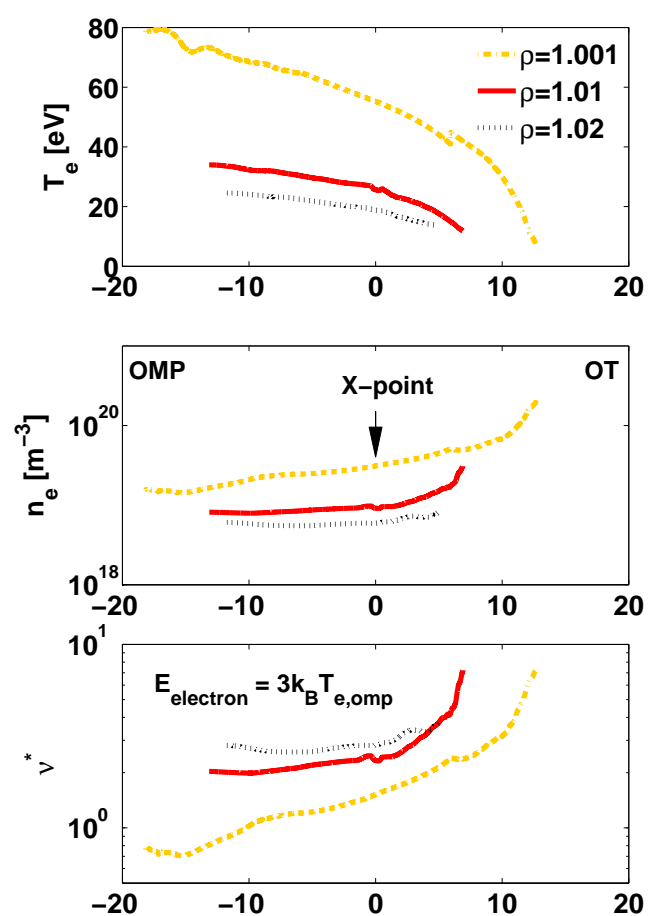

Figure 6. Theield lipedength Emlires show the electron temperature and density in the SOL plasma along the simulated field lines, from the outer midplane to the outer target. The steepest gradients are observed in the region below the X-point (field line length $\geq 0$ ), due to ionization of neutral particles. The lowest figure shows the collisionality of a suprathermal electron with energy $E=3 k_{\mathrm{B}} T_{\mathrm{omp}}$, propagating along these field lines. For the sake of reference, the scale length $L_{\mathrm{C}}$ is taken to be the connection length at $\rho=1.02$.

accompanied by a significant number of electrons at suprathermal energies. Compared to the outermost flux surfaces, the distributions at $\rho=1.001$ seem drastically different in form. The division into two components is apparently related to the temperature difference between the midplane and the target and, this being relatively small at $\rho=1.01-1.02$, such a division is scarcely visible at the outermost locations.

Close to the separatrix, the number of hot electrons is observed to increase at both targets when $m>1$, increasing also the target heat loads. In table 5 , the energy brought to the targets by suprathermal electrons $(E>30 \mathrm{eV})$ is compared to that of the thermal electrons $(E<30 \mathrm{eV})$, for the three different cases with $m=1,2,3$. The hot electron component is found to be dominating even with the thermal initial ensemble, as $71 \%$ of the outer target energy comes from this portion of electrons. Increasing the initial energy of the ensemble does not increase the energy of the thermal component at the target.

3.3.4. Identifying the collisional properties Figure 6 shows the electron temperature and density profiles together with the electron-electron collisionality along the field lines at $\rho=1.001-1.02$, on the way from the outer midplane to the outer target. At $\rho \geq 1.01$, the gradients in plasma parameters are quite small and the 
Table 6. Prompt kinetic losses, $E_{\text {kin }}$, recorded by ASCOT from 20000 electrons initialized at the outer midplane with $\rho=1.001$, compared to the theoretical energy content of a completely thermalized ensemble, $E_{\text {th }}$ (assuming $N_{\text {it }} / N_{\text {ot }}=$ $3 / 7$ based on table 4$)$.

\begin{tabular}{lclll}
\hline$T_{\mathrm{M}-\mathrm{B}}$ & $E_{\text {it,kin }}$ & $E_{\mathrm{it}, \mathrm{kin}} / E_{\mathrm{it}, \mathrm{th}}$ & $E_{\text {ot,kin }}$ & $E_{\text {ot }, \mathrm{kin}} / E_{\text {ot }, \mathrm{th}}$ \\
\hline$T_{\text {omp }}$ & $0.4 \mathrm{keV}$ & 0.004 & $4.2 \mathrm{keV}$ & 0.03 \\
$3 T_{\mathrm{omp}}$ & $101 \mathrm{keV}$ & 2.2 & $335 \mathrm{keV}$ & 2.9 \\
$5 T_{\mathrm{omp}}$ & $573 \mathrm{keV}$ & 12 & $1565 \mathrm{keV}$ & 14 \\
$7 T_{\mathrm{omp}}$ & $1326 \mathrm{keV}$ & 28 & $3708 \mathrm{keV}$ & 32 \\
\hline
\end{tabular}

collisionality remains steady until the X-point is reached. According to the simulation results, recall table 3 , the collisionality is sufficient to bring the initially suprathermal electron ensembles to the field line -averaged electron temperature. However, only at $\rho=1.01$ does the abrupt increase in collisionality close to the target seem sufficient to thermalize a notable number of electrons to the target temperature.

The profiles at $\rho=1.001$ differ from those further outside the separatrix. Above the X-point, the electron-electron collisionality is low and the background temperature remains rather high. Below the $\mathrm{X}$-point, the collisionality increases to the same level as at $\rho=1.01$, accompanied by a significant drop in the background temperature. Here, most of the electrons must be thermalized to the low target temperature, as was the case with $\rho=1.01$ also. The tremendous effect of the remaining hot electron component on the target heat load at $\rho=1.001$, recall table 5 , apparently stems from the large temperature difference between the midplane and the target.

A more detailed inspection of the hot electron component observed at $\rho=1.001$ reveals that it is only partly composed of collisionless electrons. Discarding those electrons that cool to the thermal energy from the simulation decreases dramatically the number of electrons recorded at the target. Table 6 shows the number of collisionless target electrons when initial ensembles with $m=1,3,5,7$ are used. From the initially thermal ensemble, only 7 electrons reach the plates with energy continuously above $20 \mathrm{eV}$. This is a negligible number compared to the size of the suprathermal component observed in the target distributions, recall figure 5, accounting for only few per cents of the target heat load. Significant prompt losses are observed only after increasing the initial energy above $T_{\mathrm{M}-\mathrm{B}}=3 T_{\mathrm{omp}}$. This is further depicted in figure 7 , showing prompt kinetic losses to the outer target from monoenergetic ensembles $\left(E_{\mathrm{ini}}=1-7 \times k_{\mathrm{B}} T_{\mathrm{omp}}\right)$. Although the fraction of high-energy electrons in a Maxwellian midplane plasma decreases with energy, the contribution of kinetic electrons to the target heat load increases with energy above $E_{\text {ini }}=3 k_{\mathrm{B}} T_{\text {omp }}$.

As the magnitude of the hot component at $\rho=1.001$ can not be explained by prompt losses, most of the suprathermal energy must come from the plasma at locations further downstream from the midplane. Setting a maximum of $20 \mathrm{eV}$ for the background temperature experienced by the electrons in the $m=1$ case removes the hot electron component from the target distributions, indicating that the origin of hot electrons must be somewhere around the X-point or upstream from it, where the plasma is sufficiently hot. Figure 8 shows the number of kinetic electrons recorded at the outer target, from 1000 monoenergetic electrons initialized at various locations both above and below the X-point, having initial pitch close to 1 (directed towards the outer divertor). The results indicate an energy threshold around $200 \mathrm{eV}$, below which 
the electrons do not have a chance of remaining collisionless. Higher initial energies give a finite probability for even the electrons above the $\mathrm{X}$-point to remain kinetic.

The simulations indicate that, in addition to prompt kinetic losses from the outer midplane, also the high X-point temperature contributes to the hot electron component at the target. The effect of prompt losses becomes significant only with electron ensembles having initial energy above the thermal energy. At the outermost flux surfaces with $\rho \geq 1.01$, the high collisionality above the X-point prevents significant prompt losses and, for this reason, the average target energy remains constant even with initially more energetic ensembles. For modelling purposes, these are crucial results, as the target distributions are shown to have strong dependence on the plasma properties, not only at but also in between the midplane and target plates.

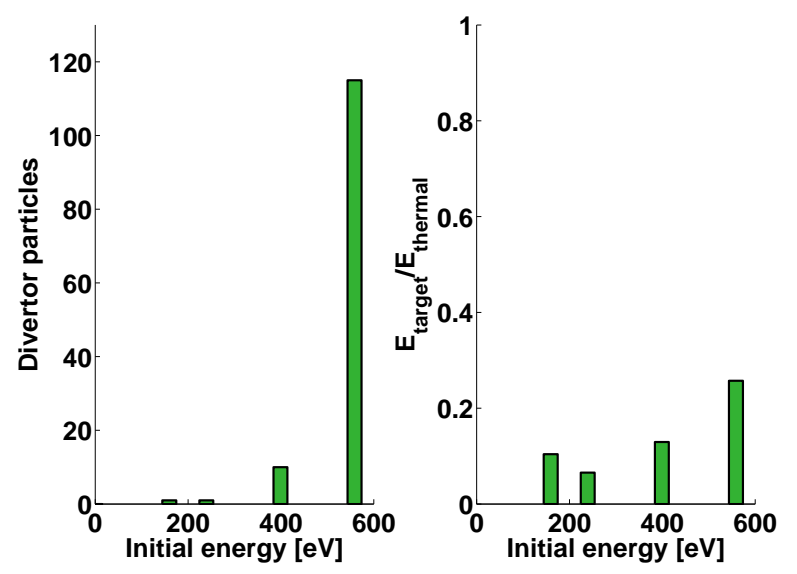

Figure 7. Left: Number of kinetic electrons (energy continuously above $20 \mathrm{eV}$ ) recorded at the outer target, from the monoenergetic ensemble of 1000 electrons with $v=v_{\|}$launched at the outer midplane $(\rho=1.001)$. Right: Contribution of kinetic electrons to the target heat load, assuming a Maxwellian midplane plasma, compared to the theoretical heat load of electrons with $E=3 k_{\mathrm{B}} T_{\mathrm{ot}} / 2$ in a rnmoletelv thermalized encemhle of the came size

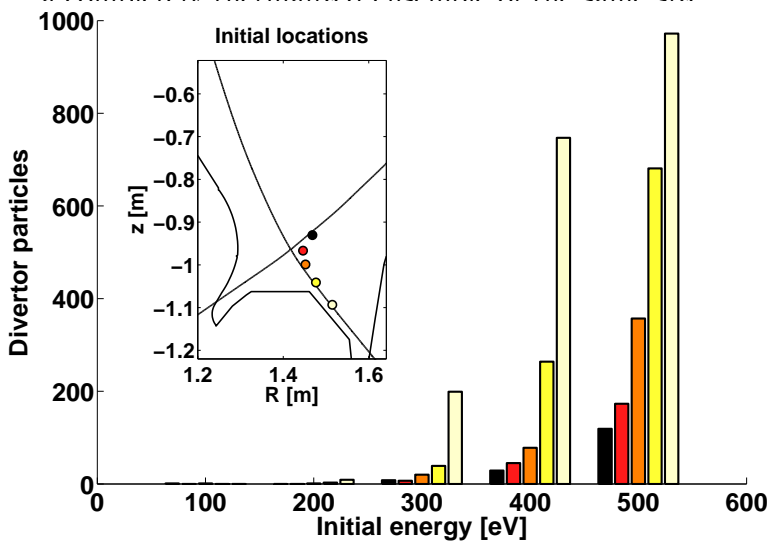

Figure 8. Kinetic electrons (energy continuously above $20 \mathrm{eV}$ ) recorded at the outer target, from the monoenergetic ensemble of 1000 electrons with $v=v_{\|}$ launched at various locations in the vicinity of the X-point $(\rho=1.001)$. 


\section{Conclusions}

The orbit-following particle simulation code ASCOT was employed in modelling an electron ensemble in a tokamak plasma, travelling through the scrape-off layer (SOL) to the divertor plates. The purpose was to investigate whether hot electrons emerging from the core plasma can propagate collisionless to the target, and how does the target heat load obtained with the Monte Carlo model agree with the fluid model for the edge plasma. A well-diagnosed ASDEX Upgrade H-mode discharge exhibiting strong temperature and density gradients in the SOL was chosen as a background for the modelling. The relevant parameters were obtained from a representative SOLPS fluid solution.

Several flux surfaces were considered, having the same collisionality on average $\left(\nu^{*} \approx 3.3(\mathrm{LFS}), \nu^{*} \approx 6.7(\mathrm{HFS})\right)$ but varying temperature and density profiles along the field line. Just outside the separatrix (at $\rho=1.001$ ), the target distributions obtained with ASCOT had a large thermal peak that was accompanied by a smaller suprathermal electron component. The hot electron component was found to be largest at the outer target, where it increased significantly the target heat load. Further outside the separatrix (at $\rho=1.01-1.02$ ), it was difficult to distinguish any thermalization to target temperature. In slight contrast, the initially suprathermal ensembles were brought to the same target distribution as the initially thermal ensemble. The statistics for the three radial positions were shown in tables 3 and 4 , and the target distributions were depicted in figures 4-5.

After considering the temperature and density profiles along the field lines, presented in figure 6 , the physics responsible for the obtained distributions could be understood. Steady collisionality along the field lines proved sufficient to bring even initially suprathermal electron ensembles close to the field line -averaged $T_{\mathrm{e}}$. Thermalization to the target temperature occured below the $\mathrm{X}$-point, provided that the divertor plasma had sufficiently high collisionality. Nevertheless, when accompanied by a large temperature gradient, even the most collisional divertor plasmas allowed hot electrons to reach the target. Just outside the separatrix, this resulted in average target energies well above those predicted by the fluid calculations. The hot component in an otherwise thermal target distribution was shown to consist of electrons remaining kinetic from various regions upstream of the target, and only a small fraction of the heat loads resulted from prompt kinetic electrons from the launching point at the outer midplane.

The ASCOT simulations presented in this paper were not, however, selfconsistent. This means that quantitative conclusions about the energy distribution close to the divertor target can not be made. In reality, the loss of suprathermal electrons to the targets would cool the divertor plasma, rendering it more collisional and more able to thermalize the electrons. In addition, non-Maxwellian energy distribution could change the electric field from that calculated by SOLPS, which would further affect the electron trajectories [13]. However, the large discrepancy obtained between the ASCOT results and the fluid model stresses the fact that kinetic effects must be considered in edge modelling.

From the results obtained one may conclude that the energy composition of electron losses is very sensitive to the distribution of the background electron density and temperature along the field lines. Close to the target, the plasma parameters are mainly affected by the ionization of neutral particles, and the SOLPS solution for the present case suggested rather strong gradients near the strike point, recall 
figure 6. In the ASCOT simulations, the abrupt change in collisionality in the divertor region was found to determine the zone from where the bulk of the losses can occur. Detailed knowledge of the magnetic geometry and of the ionization pattern in the divertor region is thus essential for realistic modelling of the background plasma and the electron losses associated with it.

\section{Acknowledgments}

This work, supported by the European Communities under the contract of Association between EURATOM/Tekes, was carried out within the framework of the European Fusion Development Agreement. The views and opinions expressed herein do not necessarily reflect those of the European Commission. The computations presented in this document have been made with CSC's computing environment. CSC is the Finnish IT centre for science and is owned by the Ministry of Education.

\section{References}

[1] Schneider R et al, 2006 Contrib. Plasma Phys. 46 3-191

[2] Taroni A et al, 1992 Contrib. Plasma Phys. 32 438-43

[3] Simonini R et al, 1994 Contrib. Plasma Phys. 34 368-74

[4] Batishchev O V et al, 1997 Phys. Plasmas 4 1672-80

[5] Fundamenski W, 2005 Plasma Phys. Control. Fusion 47 R163-R208

[6] Day M et al, 1996 Contrib. Plasma Phys. 36 419-23

[7] Heikkinen J A et al, 2001 J. Comput. Phys. 173 527-48

[8] Horacek J et al, 2003 J. Nucl. Mater. 313-316 931-5

[9] Chodura R, 1992 Contrib. Plasma Phys. 32 219-30

[10] Chankin A V et al, 2007 Nucl. Fusion 47 479-89

[11] Chankin A V et al, 2006 Plasma Phys. Control. Fusion 48 839-68

[12] Marchand R and Dumberry M, 1996 Computer Physics Communications 96 232-46

[13] Rozhansky V et al, 2006 Nucl. Fusion 46 367-82

[14] Boozer A H and Kuo-Petravic G, 1981 Phys. Fluids 24 851-9

[15] Hynönen V et al, 2007 Plasma Phys. Control. Fusion 49 151-74

[16] Kurki-Suonio T et al, 2006 Plasma Phys. Control. Fusion 48 1413-24 